\title{
Effects on metabolic markers are modified by PPARG2 and COX2 polymorphisms in infants randomized to fish oil
}

\author{
Laurine B. S. Harsløf • Camilla T. Damsgaard • \\ Lars I. Hellgren · Anders D. Andersen • \\ Ulla Vogel $\cdot$ Lotte Lauritzen
}

Received: 17 January 2014 / Accepted: 3 March 2014/Published online: 19 March 2014

(C) Springer-Verlag Berlin Heidelberg 2014

\begin{abstract}
Long-chain n-3 fatty acids (n-3 LCPUFA) improve blood pressure (BP) and lipid profile in adults and improve insulin sensitivity in rodents. We have previously shown that n-3 LCPUFA reduces BP and plasma triacylglycerol (TAG) in infants. Few studies have found effects on glucose homeostasis in humans. We explored possible effect modification by FADS, PPARG2, and COX2 genotypes to support potential effects of n-3 LCPUFA on metabolic markers in infants. Danish infants (133) were randomly allocated to daily supplementation with a teaspoon ( $\sim 5 \mathrm{~mL} /$ day) of fish oil (FO) or sunflower oil (SO) from 9 to 18 months of age. Before and after the intervention, we assessed BP, erythrocyte n-3 LCPUFA, plasma lipid profile, insulin, and glucose in addition to functional single nucleotide polymorphisms in FADS, PPARG2, and COX2. At 18 months, plasma TAG was lower in the FO compared with SO group $(p=0.014)$. This effect was modified by PPARG2-Pro12Ala, as TAG only decreased among heterozygotes. FO supplemented PPARG2
\end{abstract}

Electronic supplementary material The online version of this article (doi:10.1007/s12263-014-0396-4) contains supplementary material, which is available to authorized users.

L. B. S. Harsløf · C. T. Damsgaard · A. D. Andersen ·

L. Lauritzen $(\bowtie)$

Department of Nutrition, Exercise and Sports, Faculty

of Science, University of Copenhagen, 1958 Frederiksberg C,

Denmark

e-mail: 11@nexs.ku.dk

\section{I. Hellgren}

Department of Systems Biology, Center for Biological Sequence

Analysis, Technical University of Denmark, Lyngby, Denmark

U. Vogel

National Research Centre for the Working Environment,

Copenhagen, Denmark
Pro12Ala heterozygotes also had decreased plasma glucose compared with the SO group ( $p=0.043)$. The effect of FO on mean arterial BP at 18 months was gender dependent $(p=0.020)$ and reduced in boys only $(p=0.028)$. Diastolic BP was, however, lower among all FO supplemented homozygous COX2-T8473C variant allele carriers compared with the SO group $(p=0.001)$. In conclusion, our results confirm that FO supplementation in late infancy reduces TAG and $\mathrm{BP}$ and indicates that the effects are mediated via peroxisome proliferator-activated receptor- $\gamma$ and cyclooxygenase-2. Furthermore, FO reduced plasma glucose only in PPARG2 heterozygotes.

Keywords Long-chain n-3 polyunsaturated fatty acids . Blood pressure $\cdot$ Lipid profile · Insulin · Glucose ·

Diet-gene interaction

$\begin{array}{ll}\text { Abbreviations } \\ \text { COX-2 } & \text { Cyclooxygenase-2 } \\ \text { FA \% } & \text { Percentage of fatty acids } \\ \text { FO } & \text { Fish oil } \\ \text { LCPUFA } & \text { Long-chain polyunsaturated fatty acid } \\ \text { PPAR } \gamma & \text { Peroxisome proliferator-activated receptor- } \gamma \\ \text { PUFA } & \text { Polyunsaturated fatty acid } \\ \text { RBC } & \text { Red blood cell } \\ \text { SNP } & \text { Single nucleotide polymorphism } \\ \text { SO } & \text { Sunflower oil } \\ \text { WT } & \text { Wild-type }\end{array}$

\section{Introduction}

Numerous studies have found that consumption of $n-3$ long-chain polyunsaturated fatty acids (LCPUFA), fish, 
and fish oil positively affects various cardiovascular disease risk factors, such as plasma levels of triacylglycerol (TAG), (Harris 1997) and reduces blood pressure (BP) (Campbell et al. 2013) in adults. Furthermore, n-3 LCPUFA has been shown to improve insulin sensitivity in rodents (Poudyal et al. 2011), though these results are not supported by Cochrane review and meta-analysis of human studies (Akinkuolie et al. 2011; Hartweg et al. 2008). Our previous randomized trial found a reduction in systolic BP (SBP) and indications of a dose-dependent lowering of plasma TAG in infants who had received fish oil from 9 to 12 months of age (Damsgaard et al. 2006). A follow-up study in 6-year-old children, who as newborns had been randomized to consume formula either with or without LCPUFA, found that early intake of LCPUFA reduced BP (Forsyth et al. 2003). The small reductions in $\mathrm{BP}$ and the slightly more favorable lipid profile found in these studies may not have immediate effects on children's health, but evidence from epidemiologic studies indicates that BP levels track into adulthood (Chen and Wang 2008).

The FADS gene cluster encodes the enzymes $\Delta^{5}$ and $\Delta^{6}$ desaturase (Marquardt et al. 2000; Nakamura and Nara 2004), which are involved in the endogenous synthesis of n-3 LCPUFA (Burdge and Calder 2005). PPARG2 and COX2 encode proteins that play a role in the proposed mechanisms behind the effects of n-3 LCPUFA. The inducible cyclooxygenase-2 (COX-2) converts eicosapentaenoic acid (EPA, 20:5n-3) and arachidonic acid (AA, 20:4n-6) to prostaglandins, prostacyclins, and thromboxanes, which act as vasodilators and vasoconstrictors (Tapiero et al. 2002) and may thereby affect blood pressure. Eicosanoids and free n-3 LCPUFA also act as natural ligands for peroxisome proliferator-activated receptors (PPAR), which is a family of transcription factors involved in regulation of energy metabolism and storage (Davidson 2006). PPAR $\gamma 2$ is primarily found in adipose tissue where it regulates adipogenesis, lipid metabolism, and insulin sensitivity (Tontonoz and Spiegelman 2008).

The primary aim of the present study was to investigate possible modification by FADS, PPARG2, and COX2 genotypes of effects of n-3 LCPUFA on metabolic markers. We also investigated the consistency of our previous findings of reduction in blood pressure and plasma triacylglycerol by fish oil supplementation (Damsgaard et al. 2006) and assessed potential effects of n-3 LCPUFA on glucose homeostasis. The present study included more participants than our earlier trial, a longer intervention period, and a control group, in order to ensure that the effects found were due to n-3 LCPUFA and not merely to oil or PUFA as such.

\section{Subjects and methods}

Study design and subjects

The EFiON (Essentielle Fedtsyrer i OvergangskosteN) study was a randomized, controlled, and double-blinded trial in which infants were allocated to a daily oil supplement from 9 to 18 months of age (NCT 00631046 at ClinicalTrials.gov). The study was conducted at Department of Nutrition, Exercise, and Sports, University of Copenhagen from January 2008 to March 2009, and it was approved by the Scientific Ethics Committees of the Capital Region of Denmark (no. H-A-2007-0088) and registered at ClinicalTrials.gov as NCT 00631046. The study design and methods are described in detail by Andersen et al. (2011).

In short, families with infants in the Capital Region of Denmark were invited to participate through the National Danish Civil Registry and were sent written information about the study. Parents who wished to participate were invited to individual information meetings where they gave written informed consent. Eligible infants were healthy singletons, born $>37$ th week of gestation and with appropriate weight for gestational age. Infants were excluded if they had previously received fish oil supplements, chronic diseases, or received medication that could influence growth and/or food intake.

\section{Intervention}

The infants were randomized to receive a teaspoon (approximately equivalent to $5 \mathrm{~mL} /$ day) of fish oil (FO, rich in eicosapentaenoic acid (EPA; 20:5n-3) and docosahexaenoic acid (DHA; 22:6n-3) but without vitamins) or a teaspoon of sunflower oil (SO, rich in linoleic acid; 18:2n6) (both kindly donated by Axellus A/S). To avoid excessive energy intake, parents were instructed to substitute some of the fat in the infant's diet with the oil provided. The oils were provided in ten dark bottles of similar appearance $(1.5 \mathrm{~L}$ in total) and all bottles, empty or containing oil leftovers were returned for the assessment of compliance at the 18 months examination. The group allocation was performed blinded and based on a computer-generated block randomization with varying block sizes of 4, 6, 8, and 10 (www.randomization.com) as described elsewhere (Andersen et al. 2011).

\section{Measurements}

Detailed questionnaires were completed before the examinations at 9 and 18 months, and information on the average food intake of the infants was obtained through 
validated pre-coded food diaries completed by the parents on seven consecutive days prior to the two examinations (Gondolf et al. 2012). All measurements were taken in the presence of the infants' parents.

The anthropometric measurements were taken by trained investigators as described in detail by Andersen et al. (2011). Recumbent length was measured to the nearest millimeter on a wooden measuring board (Force Technology, Brøndby, Denmark). A total of three measurements were taken, and the mean value of the measurements was used. The infant's weight was measured on a pediatric infant scale (Sartorius IP65; Bie \& Berntsen AS, Rødovre, Denmark), which measured the weight 40 times for a few seconds and provided the average of all the measurements. If the infant weighed $\geq 12 \mathrm{~kg}$, the infant was weighed with a parent on an adult scale (Lindeltronic 8000; Samhall Lavi AB, Kristianstad, Sweden) with subsequent subtraction of parent weight.

Blood pressure and heart rate

$\mathrm{BP}$ and heart rate (HR) were measured, while the infant sat on the parent's lap. To ensure a minimum of movement, one examiner distracted the infant with toys. Parents of infant's that moved too much were instructed to gently hold the infant's hand to reduce movement of the arm where BP was measured. At 9 months, BP was measured using an automated oscillometric device (model $506 \mathrm{~N}$, Criticare Systems Inc., Waukesha, WI, USA) and a small cuff fitting arm circumferences of $10-19 \mathrm{~cm}$. At 18 months, $26 \%$ of the infants had their BP measured on the same device, but a different automated oscillometric device (Spot Vital Signs LXi, Welch Allyn, Skaneateles Falls, NY, USA) was used on $61 \%$ of the infants due to break down of the original apparatus. The break down also resulted in a lack of BP assessments in $13 \%$ of the infants at 18 months. We aimed to measure BP three times during approximately $15 \mathrm{~min}$, and this was fulfilled for 65 and $57 \%$ of the infants at 9 and 18 months, respectively. Four measurements were taken from 33 to $30 \%$ of the infants at 9 and 18 months, respectively, whereas only 1-2 blood pressure measurements were taken for the remaining infants. Recordings deviating $>3 \mathrm{SD}$ from the overall mean of all measurements from all infants were deselected resulting in the exclusion of $1-5 \%$ of the individual measurements of SBP, diastolic BP (DBP), mean arterial BP (MAP), and HR, at both examinations. The mean of the remaining $\mathrm{BP}$ and $\mathrm{HR}$ measurements was used in the statistical analyses. The mean intra-individual coefficients of variance (CV) after exclusion of blood pressure measurements were 10, 17, and $13 \%$ for SBP, DBP, and MAP, respectively.
Blood sample

Venous blood sampling was successfully achieved in 120 infants at baseline and 128 infants at 18 months of age. Blood sampling was successful for 115 infants on both occasions. Parents were instructed to apply an eutectic mixture of local anesthetics patches (AstraZeneca AB) to anesthetize the skin prior to blood sampling. Parents were instructed to prevent their child from eating at least $2 \mathrm{~h}$ prior to blood sampling and were asked when and what the infant had consumed at the last meal prior to the blood sampling. The nutrient composition of the meal was approximated using Dankost (vers. 3000, Dankost Ltd., Copenhagen, Denmark). Mean fasting time $( \pm S D)$ was approximately $157 \pm 47$ and $150 \pm 33 \mathrm{~min}$, with the exception of three infants who had fasted 14-16 $\mathrm{h}$ prior to the examination at 18 months.

RBC fatty acid analyses

Erythrocytes (RBC) were isolated from heparinized blood samples, washed, and kept at $-80{ }^{\circ} \mathrm{C}$ for later analysis, as described elsewhere (Lauritzen et al. 2004). The content of specific fatty acids was calculated as area $\%$ of the specific fatty acid relative to the total identified chromatogram area (FA \%), which is almost equivalent to \% by weight.

\section{Plasma lipid profile}

Concentrations of total, LDL, and HDL-cholesterol, and TAG in heparinized plasma, were determined using an automated, enzymatic, colorimetric assay on ABX Pentra 400 (HORIBA ABX, Montpellier, France). Analytical intra-serial variations (CV \%) were as follows: $1.5 \%$ for total cholesterol, $2.6 \%$ for LDL-c, $1.8 \%$ for HDL-c, and $3.0 \%$ for TAG.

\section{Plasma glucose, insulin, and HOMA}

Glucose concentration was determined in EDTA whole blood immediately after blood sampling on a HemoCue analyzer (HemoCue Danmark, Vedbaek, Denmark), and the equivalent plasma values were obtained by multiplying with 1.11 (D'Orazio et al. 2005). Plasma insulin was determined in heparinized plasma by automated assays on an Immulite 1000 (Siemens Healthcare Diagnostics, Ballerup, Denmark).

Plasma insulin concentrations below the detection limit $(<12 \mathrm{pmol} / \mathrm{L})$ were defined as $6 \mathrm{pmol} / \mathrm{L}(n=23)$. HOMAIR was calculated as plasma glucose $(\mathrm{mmol} / \mathrm{L}) \times$ plasma insulin (mIU/L)/22.5 (Cutfield et al. 2003). Insulin concentrations were converted from $\mathrm{pmol} / \mathrm{L}$ to $\mathrm{mIU} / \mathrm{L}$ by dividing with 6 (Heinemann 2010). 
Selection of SNPs and genotyping

We selected three FADS SNPs for genotyping (rs1535, rs174575, and rs174448), which each tag 17, 6, and 5 other SNPs in the 100-kb genomic region of the FADS1, FADS2, and FADS3 gene cluster, as described elsewhere (Harslof et al. 2013). The following SNPs were also genotyped: PPARG2-Pro12Ala (rs1801282) (as described by Vogel et al. 2007a) that has been shown to reduce transcription of target genes (Masugi et al. 2000); COX2-C8473T (rs5275) (Campa et al. 2004) and COX2 A-1195G (rs689466) (Vogel et al. 2007b), which have been shown to up- and down-regulate the COX-2 response, respectively, among variant allele carriers (Sanak et al. 2005; Zhang et al. 2005).

DNA was isolated from buffy coat preparations from EDTA blood, which was kept at $-80{ }^{\circ} \mathrm{C}$ until DNA extraction, performed as described by Miller et al. (Miller et al. 1988). DNA dissolved in TE buffer was kept at $-20{ }^{\circ} \mathrm{C}$ until genotyping. Genotyping was performed using approximately $20 \mathrm{ng}$ DNA in a total volume of $6 \mu \mathrm{L}$ containing $1 \times$ Mastermix (Life Technologies, Nærum, Denmark), $100 \mathrm{nmol} / \mathrm{L}$ probes, and $900 \mathrm{nmol} / \mathrm{L}$ primers. A test run of minimum 24 samples was genotyped using realtime PCR to identify controls with known genotypes. All samples were genotyped using endpoint PCR. Controls were included in each run, and genotyping was repeated in a random $14 \%$ subset which yielded $100 \%$ identical genotypes. Genotypes were analyzed in $88 \%(n=117)$ of the infants, and the seven SNPs were genotyped with a success rate of $100 \%$. All the included polymorphisms were in Hardy-Weinberg equilibrium $(p>0.05)$.

\section{Statistical analysis}

Statistical analyses were performed using Stata 12.0 (Stata Institute), and significance was established at $p<0.05$. Data are presented as mean \pm SD or median and interquartile range as appropriate. The distribution of three outcome variables-plasma TAG, plasma insulin, and HOMA-was skewed to the right and was therefore logtransformed before statistical analysis. Only participants with available outcome data at both examinations were included in the analyses (complete case analysis). For each outcome, two ANCOVA models were used: a baselineadjusted model and a full confounder adjusted model. Stepwise backward model reduction was used to remove interaction terms and covariates from the confounder adjusted model. Covariates that were likely to affect the outcome $(p<0.10)$ were kept in the model. For all outcomes, the full model initially included baseline measurement, breastfeeding during the intervention (yes/no), birth weight $(\mathrm{g})$, and an intervention group $(\mathrm{FO} / \mathrm{SO}) \times$ gender (girl/boy) interaction term. Lipid profile outcomes, insulin, glucose, and HOMA were adjusted for BMI $\left(\mathrm{kg} / \mathrm{m}^{2}\right)$, time since last meal (minutes), and energy content of last meal $(\mathrm{kJ})$. Blood pressures and heart rate were also adjusted for length $(\mathrm{cm})$, average number of sun light hours during 18 month BP assessment, and type of oscillometric device. Possible intervention modifying effects of FADS genotype were investigated by including an intervention group $\times$ number of DHA-increasing alleles interaction term. Number of DHA-increasing alleles was derived in accordance with our previous findings, with the rs 1535 SNP coded opposite of rs174575 and rs174448 (Harslof et al. 2013). Effect modification by PPARG2 and COX2 genotype was investigated by including an intervention group $\times$ SNP interaction term in the final model coded as a categorical explanatory variable, with homozygocity for the major allele (WT) coded as 1, heterozygocity coded as 2 , and homozygocity for the minor allele (variant) coded as 3. Only one child was homozygote for the minor allele of PPARG2 and was therefore excluded. Paired t tests were used to assess within group changes. Correlations between RBC EPA or DHA and oil consumption or outcome variables were assessed with Pearson's product moment correlation.

\section{Results}

Overall, the randomization was successful, with comparable baseline characteristics being found in the two groups (Table 1). Median plasma insulin at 9 months was lower in the FO group, mainly due to a larger spread. The PUFA intake was slightly higher in the FO group, and fish intake was also somewhat skewed with a higher intake in the FO group and more infants who had not been introduced to fish in the SO group, but the RBC fatty acid composition was similar (Andersen et al. 2011).

The median intake of intervention oil was 3.8 (3.2-4.2) g/day, which provided around $630 \mathrm{mg} /$ day EPA and $620 \mathrm{mg} /$ day of DHA (Andersen et al. 2011). Eicosapentaenoic acid and DHA in RBC were significantly increased in the FO group, EPA by 5.5 (4.1-7.4) FA $\%$ and DHA by $4.8 \pm 0.3 \mathrm{FA} \%$, relative to the changes in SO group (EPA: $0.5(0.0-1.1)$ FA \%, DHA: $1.0 \pm 0.3$ FA \%. $p<0.001$ for both fatty acids)) (Andersen et al. 2011). The estimated daily intake of intervention oil was linearly associated with RBC EPA in the FO group at 18 months (Fig. 1).

A potential effect of $F A D S$ SNPs on DHA status was investigated by including the FADS SNPs individually in the model, entered all together in the model, or combined 
Table 1 Baseline characteristics of the study population
Values are mean \pm SD or median (25th-75th quartiles) (n) unless stated otherwise

$F O$ fish oil, $M M$ homozygote major allele carriers, $\mathrm{Mm}$ heterozygotes, $\mathrm{mm}$ homozygote minor allele carriers, and $S O$ sun flower oil

a Plasma insulin concentrations below the detection limit $(<12 \mathrm{pmol} / \mathrm{L})$ were defined as $6 \mathrm{pmol} / \mathrm{L}(n=23)$

${ }^{b}$ Only one child was homozygote for the minor allele of PPARG2 and was therefore excluded from the statistical analyses

\begin{tabular}{|c|c|c|}
\hline Birth characteristics and breastfeeding & FO group & SO group \\
\hline Gender $[n, \mathrm{~F}: \mathrm{M}(\% \mathrm{M})]$ & $33: 28(46)$ & $29: 43(60)$ \\
\hline Gestational length (wk) & $40.0 \pm 1.5(57)$ & $40.0 \pm 1.4(69)$ \\
\hline Birth weight $(\mathrm{kg})$ & $3.5 \pm 0.4(61)$ & $3.6 \pm 0.5(71)$ \\
\hline Birth length (cm) & $51.9 \pm 1.9(61)$ & $52.3 \pm 2.3(71)$ \\
\hline Breastfeeding—duration full (mo) & $4.0(4.0-5.0)(61)$ & $4.0(4.0-5.0)(71)$ \\
\hline Breastfed at 9 months (\%yes) & 52 & 49 \\
\hline \multicolumn{3}{|l|}{ Anthropometry } \\
\hline Age (mo) & $9.1 \pm 0.3(61)$ & $9.1 \pm 0.3(72)$ \\
\hline Length (cm) & $72.1 \pm 2.4(61)$ & $72.5 \pm 2.4(72)$ \\
\hline BMI $\left(\mathrm{kg} / \mathrm{m}^{2}\right)$ & $17.9 \pm 1.5(61)$ & $17.6 \pm 1.4(72)$ \\
\hline \multicolumn{3}{|l|}{ Metabolic markers } \\
\hline Plasma triacylglycerol (mmol/L) & $1.21(0.86-1.87)(57)$ & $1.41(0.93-2.10)(62)$ \\
\hline Plasma total cholesterol (mmol/L) & $4.04 \pm 0.83(57)$ & $4.04 \pm 0.90(62)$ \\
\hline Plasma LDL-cholesterol (mmol/L) & $2.32 \pm 0.66(57)$ & $2.34 \pm 0.77(62)$ \\
\hline Plasma HDL-cholesterol (mmol/L) & $1.10 \pm 0.25(57)$ & $1.06 \pm 0.25(62)$ \\
\hline Plasma insulin ${ }^{\mathrm{a}}(\mathrm{pmol} / \mathrm{L})$ & $31(15-64)(57)$ & $39(22-61)(60)$ \\
\hline Plasma glucose $(\mathrm{mmol} / \mathrm{L})$ & $5.1 \pm 0.7(58)$ & $5.3 \pm 0.6(63)$ \\
\hline HOMA & $1.0(0.5-2.4)(57)$ & $1.5(0.8-2.3)(60)$ \\
\hline Systolic blood pressure (mmHg) & $111 \pm 13(59)$ & $112 \pm 10(59)$ \\
\hline Diastolic blood pressure (mmHg) & $66 \pm 9(59)$ & $64 \pm 7(72)$ \\
\hline Mean arterial pressure $(\mathrm{mmHg})$ & $84 \pm 9(58)$ & $83 \pm 8(72)$ \\
\hline Heart rate $(\mathrm{bpm})$ & $99 \pm 23(60)$ & $103 \pm 20(72)$ \\
\hline \multicolumn{3}{|l|}{ Daily dietary intake } \\
\hline Energy intake $(\mathrm{kJ})$ & $3,260 \pm 1,134(61)$ & $3,150 \pm 831(70)$ \\
\hline Protein intake (E \%) & $12.4 \pm 1.9(61)$ & $12.6 \pm 2.1(70)$ \\
\hline Carbohydrate intake (E \%) & $50.5 \pm 4.6(61)$ & $51.4 \pm 6.2(70)$ \\
\hline Fat intake $(\mathrm{E} \%)$ & $37.2 \pm 4.4(61)$ & $36.0 \pm 5.9(70)$ \\
\hline SFA & $12.8 \pm 3.9(61)$ & $14.0 \pm 3.9(70)$ \\
\hline MUFA & $12.6 \pm 2.9(61)$ & $12.1 \pm 2.6(70)$ \\
\hline PUFA & $7.7(5.3-9.2)(61)$ & $6.1(4.9-7.8)(70)$ \\
\hline n-3 PUFA & $0.9(0.7-1.2)(61)$ & $0.9(0.7-1.0)(70)$ \\
\hline n-6 PUFA & $5.8(3.8-7.6)(61)$ & $4.8(3.7-6.0)(70)$ \\
\hline n-6/n-3PUFA & $6.2(4.4-8.0)(61)$ & $5.8(4.4-7.2)(70)$ \\
\hline Fish introduced at 9 months [yes:no (\%yes)] & $56: 5(92)$ & $54: 16(74)$ \\
\hline Fish intake $(\mathrm{g})$ & $10.6(3.4-14.7)(50)$ & $6.3(2.9-12.5)(62)$ \\
\hline \multicolumn{3}{|l|}{ Erythrocyte FA composition } \\
\hline EPA 20:5n-3 (FA \%) & $0.92(0.66-1.32)(57)$ & $0.82(0.63-1.15)(63)$ \\
\hline DHA 22:6n-3 (FA \%) & $6.67 \pm 1.83(57)$ & $6.69 \pm 1.54(63)$ \\
\hline \multicolumn{3}{|l|}{ Genotype distribution (MM:Mm:mm) } \\
\hline PPARG2-Pro12Ala (rs1801282) & $44: 10: 1^{b}$ & $52: 10: 0$ \\
\hline COX2-C8473T (rs5275) & $23: 25: 7$ & $29: 26: 7$ \\
\hline COX2 A-1195G (rs689466) & $37: 16: 2$ & $43: 17: 2$ \\
\hline
\end{tabular}

to one variable with number of alleles increasing DHA status that were significant at 9 months (Harslof et al. 2013). None of the FADS SNPs had an effect on DHA status at 18 months (Figure S1) and were therefore not included in any of the analyses with metabolic markers as outcome.
Blood pressure and heart rate

Systolic blood pressure was affected by COX2-C8473T genotype independently of the intervention as homozygous variant allele carriers (having a theoretically increased COX-2 activity) tended to have lower SBP at 18 months 


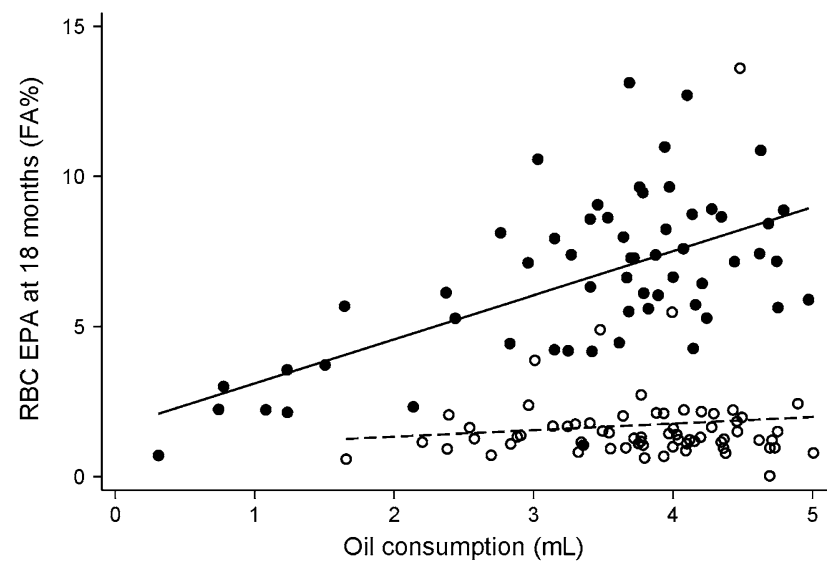

Fig. 1 Correlation between the estimated average daily consumption of intervention oil and eicosapentaenoic acid (EPA) in erythrocytes (RBC) at 18 months. The black dots and solid line give values from the fish oil group $(r=0.61, p<0.001, n=59)$, and the white dots and dashed line give values from the sunflower oil group $(r=0.09$, $p=0.466, n=66$ )

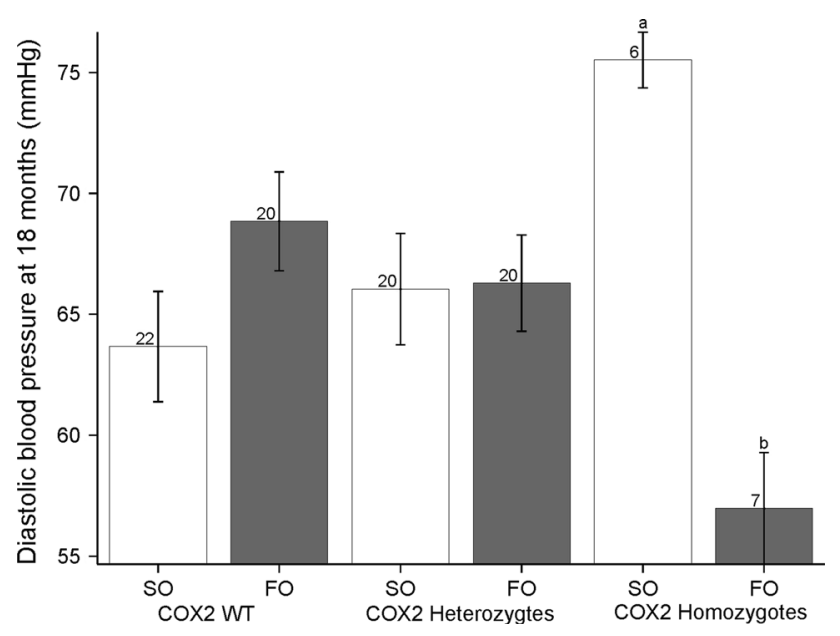

Fig. 2 Diastolic blood pressure at 18 months by $C O X 2-\mathrm{C} 8473 \mathrm{~T}$ genotype and group. Significant difference between the fish oil (FO) and sunflower oil (SO) supplemented groups adjusted for baseline is indicated with different letters $(p=0.001)$. Values on bars are $n$

compared with homozygous WT $(\beta=-7 \mathrm{mmHg}$, $p=0.064$ adjusted for baseline, gender, hours of sun light and BP device). For DBP, there was a significant interaction between intervention group and COX2-C8473T genotype $(p<0.001)$, with DBP being $18 \mathrm{mmHg}$ lower in the FO group versus the SO group only in homozygous variant carriers $(p=0.001)$ (Fig. 2). A combined analysis including both PPARG2 and COX2-C8473T genotypes revealed that the FO supplemented infants, who were homozygous WT for both PPARG2 and COX2-C8473T (i.e., having a theoretically normal COX-2 and PPAR $\gamma$ activity), had higher DBP compared with the SO group $(p=0.012)$. There was also a tendency for effect modification by COX2-C8473T genotype on MAP ( $p=0.098$, adjusted for gender), with MAP being borderline significantly lower in the FO group versus the SO group in homozygous variant carriers $(\beta=-13 \mathrm{mmHg}, p=0.051$, adjusted for gender). Furthermore, PPARG2 heterozygote infants (having theoretically lower PPAR $\gamma$ activity) had higher MAP compared with homozygous WT ( $\beta=8 \mathrm{mmHg}, p=0.010, n=86)$ independent of the intervention.

There was no difference for either SBP or DBP between groups at 18 months, but there was effect modification by gender for MAP (Table 2). Mean arterial pressure was lower in the FO compared with SO group among the boys, whereas there was no difference among the girls. This finding was confirmed by a positive correlation between RBC DHA at 18 months and the change in MAP in boys only (Fig. 3). The changes in HR tended to be significantly different between the groups (Table 2), and this correlated positively with RBC DHA at 18 months (Fig. 4).

Lipid profile and plasma insulin and glucose

Following the intervention, plasma TAG was lower in the FO group compared with the SO group (Table 2). Plasma TAG was negatively correlated with RBC DHA at 18 months (Fig. 5), but there was no correlation with erythrocyte levels of EPA $(r=-0.14, p=0.120$, $n=127$ ) (not shown). PPARG2 genotype tended to modify the effect of the intervention on plasma TAG $(p=0.055)$ as this only decreased in the FO group compared with the SO group among PPARG2 heterozygotes, who are known to have genetically determined lower PPAR $\gamma$ activity (Fig. 6a). Total cholesterol, LDL-cholesterol, and HDL-cholesterol did not differ between the groups at 18 months (Table 2). The intervention did not result in any differences in plasma insulin, glucose, or HOMA between the two groups. Neither plasma insulin nor plasma glucose were correlated with RBC DHA at 18 months $(r=-0.12$ and $-0.12 ; p=0.176$ and 0.175 , respectively). However, plasma glucose among PPARG2 heterozygotes only decreased in the FO group $(p=0.043)$ (Fig. 6b).

\section{Discussion}

Few studies have investigated the effects of fish oil on metabolic markers in infancy, and this is the first study to include genotypes of FADS, PPARG2, and COX2 genes. We found that n-3 LCPUFA supplementation reduced BP, TAG, and glucose, and the effects seemed to be mediated via COX-2 and PPAR $\gamma 2$. As the impact of FADS genotype 


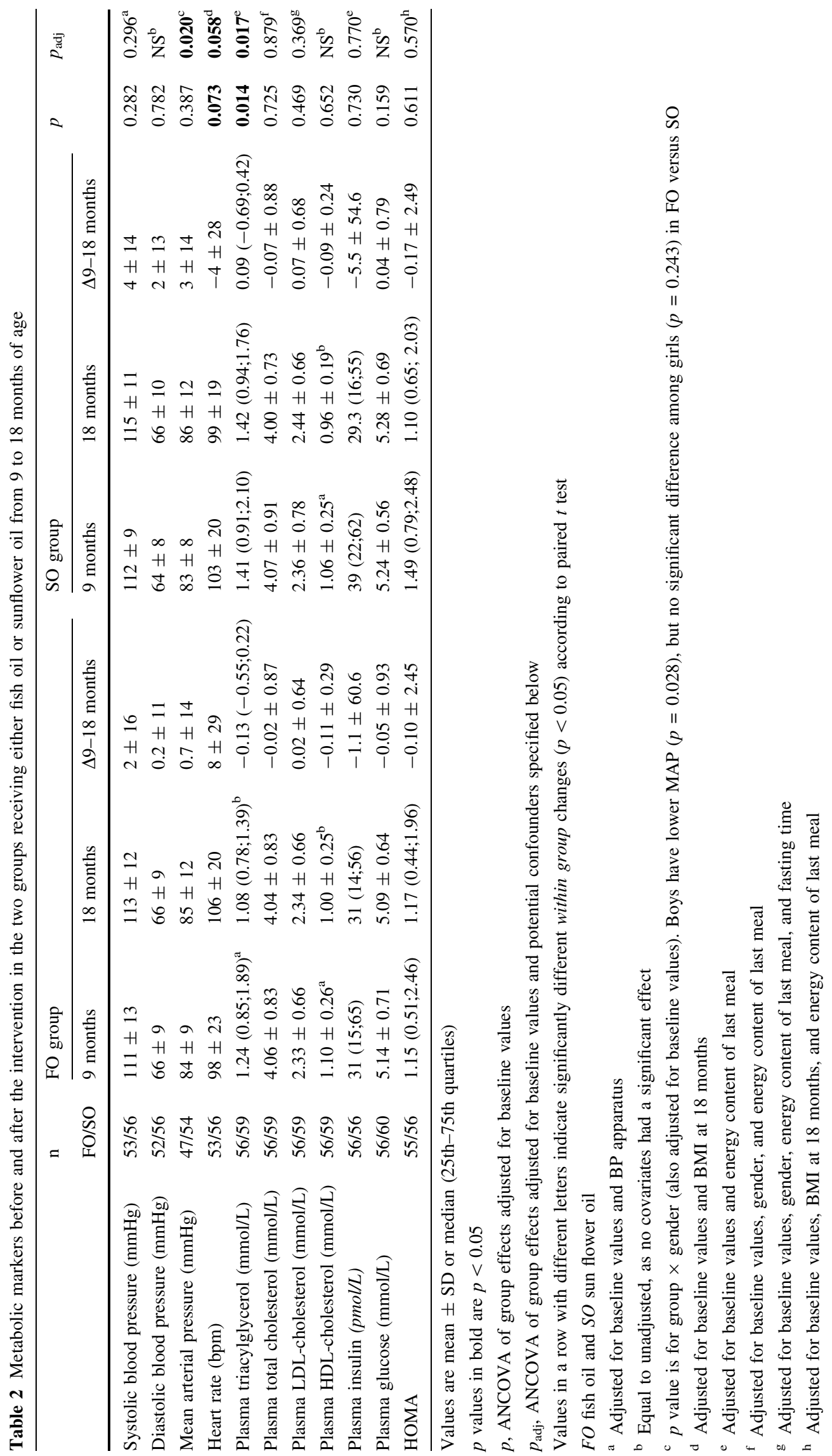




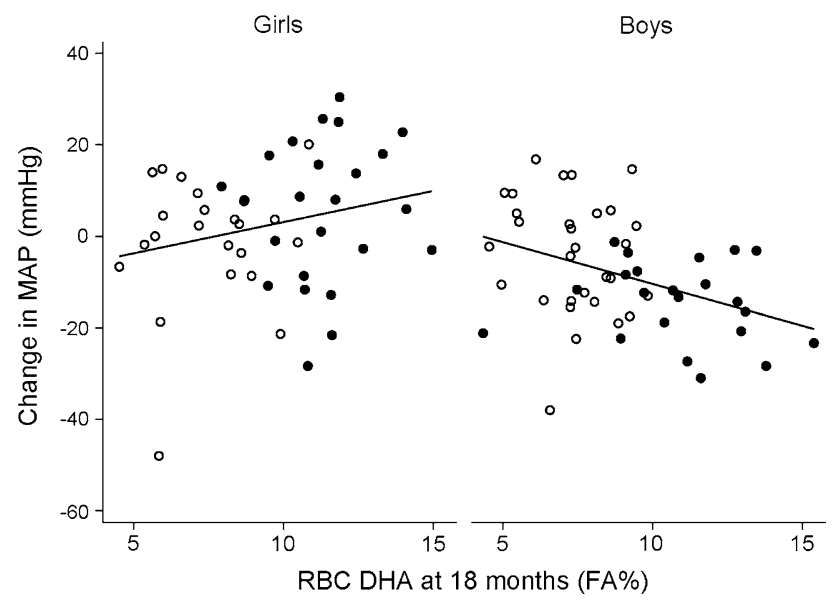

Fig. 3 Correlation between the erythrocyte levels of docosahexaenoic acid (RBC DHA) at 18 months and change in mean arterial pressure (MAP) during the intervention. The black and white dots give values from the fish oil and sunflower oil group, respectively. Regression lines: girls $(r=0.23, p=0.12, n=47)$ and boys $(r=$ $-0.38, \quad p=0.005, n=52)$. Baseline-adjusted MAP values at 18 months among girls were as follows: $86.2 \pm 2.7$ and $82.3 \pm 2.8$, and among boys: $82.5 \pm 2.1$ and $89.7 \pm 1.8$ in the FO and SO group, respectively

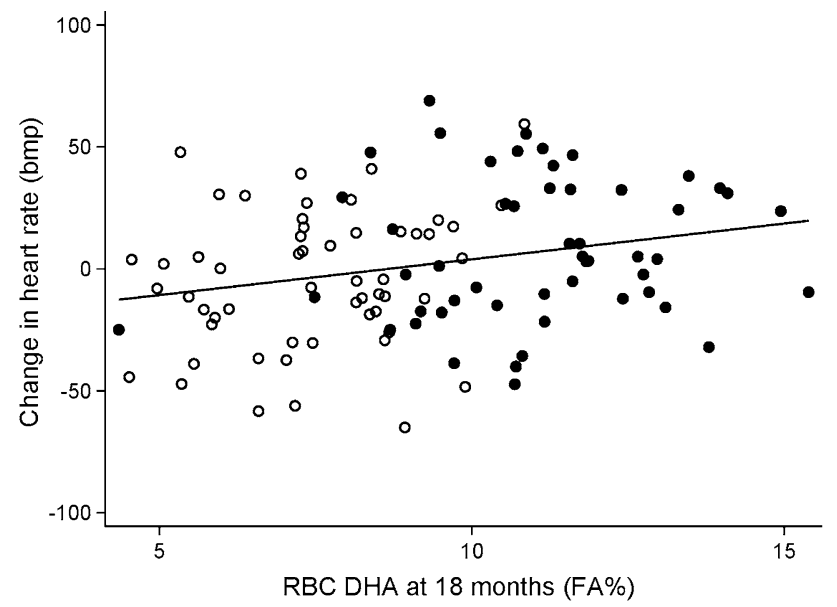

Fig. 4 Correlation between erythrocyte levels of docosahexaenoic acid (RBC DHA) and change in heart rate during the intervention. The black and white dots give values from the fish oil and sunflower oil group, respectively. The regression line $(r=0.26, p=0.008$, $n=107)$

is mediated via its effect on PUFA status, we chose not to include the individual FADS SNPs in the outcome analyses, but focused on PPARG2 and COX2, which encodes proteins involved in the proposed mechanism of action of n-3 LCPUFA on the metabolic markers.

We have previously shown that the FADS SNPs affected DHA status at 9 months of age and that the SNPs can be combined into one variable with numbers of DHAincreasing alleles (Harslof et al. 2013). In the present study, we observed a borderline significant interaction between

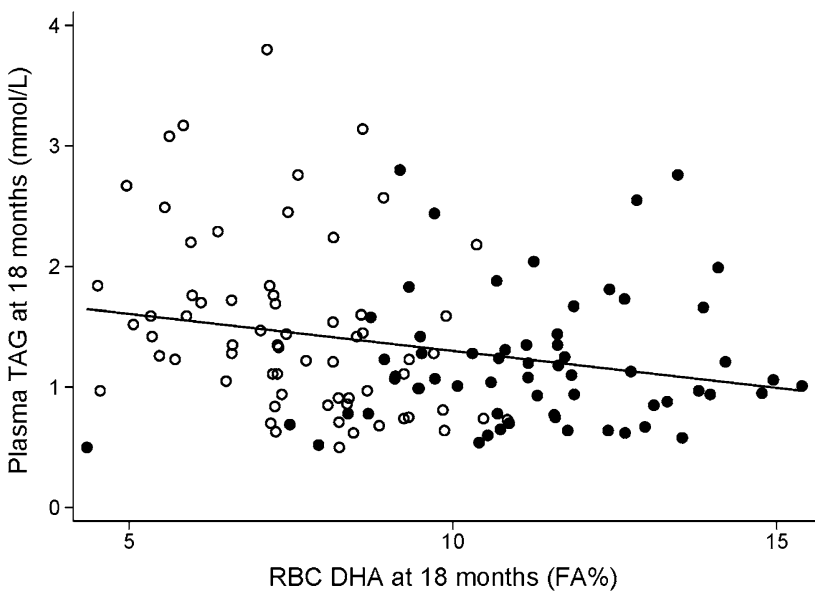

Fig. 5 Correlation between erythrocyte levels of docosahexaenoic acid (RBC DHA) and plasma triacylglycerol (TAG) at 18 months. The black and white dots give values from the fish oil (FO) and sunflower oil (SO) group, respectively. Regression line: $r=-0.24$, $p=0.007, n=127$. Regression lines for the $\mathrm{SO}$ and $\mathrm{FO}$ groups alone were $r=-0.35, p=0.003, n=67$ and $r=0.11, p=0.412$, $n=60$, respectively (not shown)

the FO intervention and number of DHA-increasing alleles, although there were only a few infants in the low and high category of this combined FADS SNP variable. However, the high dose of FO tended to overrule the endogenous capacity for n-3 LCPUFA synthesis as the effect of FADS genotype on EPA and DHA was insignificant compared with the group difference.

The present study supports our previous finding that $n-3$ LCPUFA can reduce plasma TAG in infants (Damsgaard et al. 2006). Based on the interaction with PPARG2, this effect appears to be mediated via PPAR $\gamma 2$, which is primarily found in adipose tissue where it regulates adipocyte differentiation and metabolism (Ahmadian et al. 2013; Tontonoz and Spiegelman 2008). Both n-3 LCPUFA and eicosanoids can activate PPAR $\gamma$, which then promotes lipid uptake and storage in adipose tissue (Tontonoz and Spiegelman 2008). The variant of the PPARG2 Pro12Ala polymorphism has been shown to reduce transcription of target genes (Masugi et al. 2000), so we expected the greatest effects on plasma TAG among those who were homozygous WT for PPARG2. Instead, we found that plasma TAG decreased in the FO group only in PPARG2 heterozygotes. This is in line with the findings of Lindi et al. (2003), who observed a greater decrease in serum TAG among variant allele carriers following 3 months of FO supplementation, but only when dietary intake of total fat or saturated fatty acids was below 37 and $10 \mathrm{E} \%$, respectively. We did not find an increase in plasma cholesterol in the FO relative to the SO group, which is in contrast to the findings of our earlier study (Damsgaard et al. 2006). However, the control group of this previous 

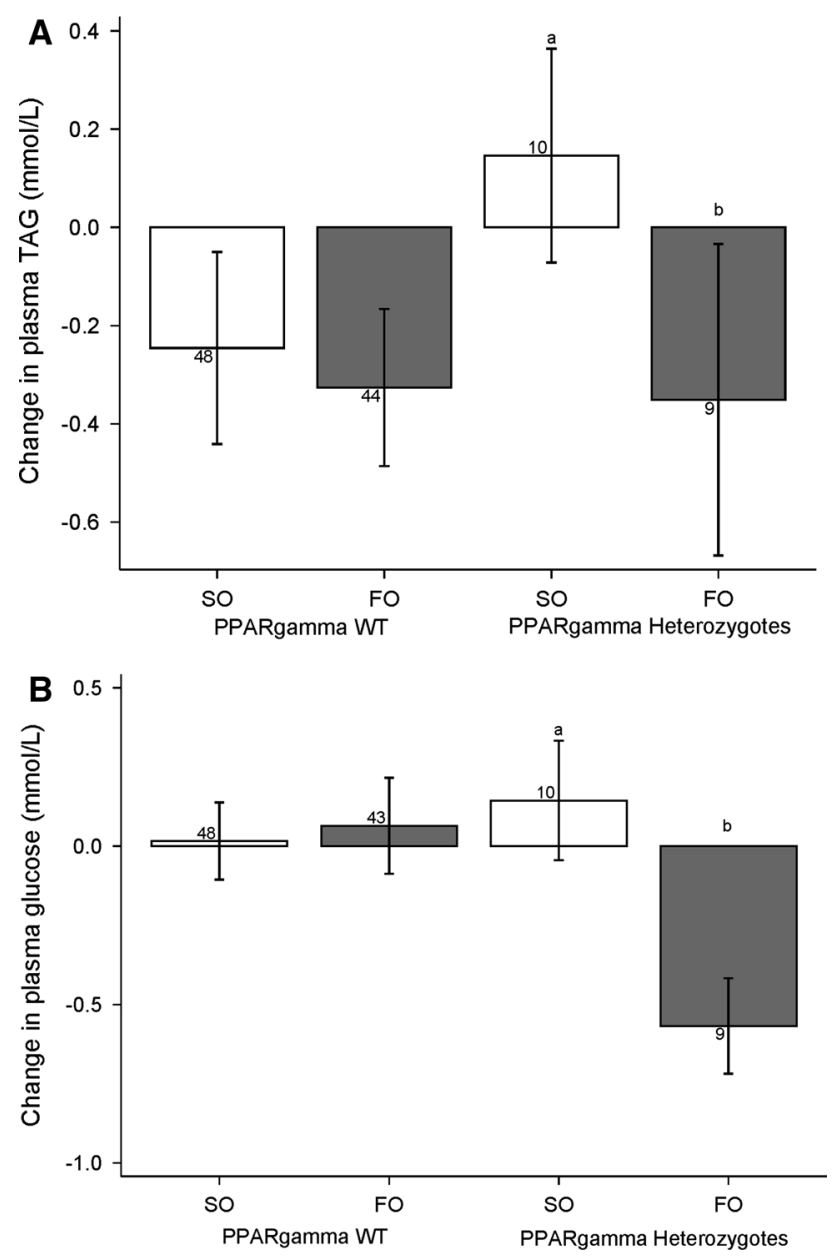

Fig. 6 Change in plasma TAG (a) and plasma glucose (b) during the intervention by PPARG2-Pro12Ala genotype and group. $p$ values are shown for significant differences between the fish oil (FO) and sunflower oil (SO) supplemented groups adjusted for baseline and energy intake (TAG only) in linear regression models with either plasma TAG (log-transformed) $(p=0.008)$ or plasma glucose at 18 months as outcome $(p=0.043)$. Values on bars are $n$

study did not receive any oil and the observed increase could thus have been due to a difference in fat intake.

PPARG2 is also involved in controlling genes regulating glucose homeostasis (Ahmadian et al. 2013), and the variant of the PPARG2 Pro12Ala polymorphism has been associated with a reduced risk of diabetes (Stumvoll and Haring 2002). This is in line with the findings of a study in 571 adults where postprandial plasma glucose was lower among female variant allele carriers of PPARG2 Pro12Ala (Ylonen et al. 2008). That FO supplementation only seems to exert an effect on plasma glucose among PPARG2 heterozygotes may explain why previous studies in humans have been unable to show an effect of FO on glucose homeostasis and insulin resistance. We found no effect of FO on glucose homeostasis and HOMA, which is in line with findings by Pedersen et al. (2010) in teenage boys and findings from studies in adults (Egert et al. 2008; Giacco et al. 2007). In contrast, other studies in adults have observed improved insulin sensitivity as a consequence of fish intake or FO supplementation (Abete et al. 2008; Ramel et al. 2008).

We found lower DBP in the FO group compared with SO group, but only among homozygotes for the minor COX2 C8473T allele, who theoretically have increased COX-2 activity. A possible mechanism by which $n-3$ LCPUFA may affect BP is through conversion to eicosanoids as EPA-derived eicosanoids are generally less potent vasoconstrictors than the corresponding eicosanoids from AA (Tapiero et al. 2002). Individuals with increased COX2 activity may, therefore, produce more of the less potent eicosanoids when intake of $n-3$ LCPUFA is increased and more EPA becomes available relative to AA. The present findings on BP are in line with the results of our earlier study with FO supplementation versus no supplementation from 9 to 12 months of age (Damsgaard et al. 2006). Further analysis of the data from this earlier study has revealed that the reduction in SBP was also only significant among boys (unpublished data). A lower SBP and DBP was also seen in teenage boys after supplementation with FO compared with vegetable oil for 16 weeks (Pedersen et al. 2010), and numerous studies have demonstrated a BP lowering effect of FO in adults (Campbell et al. 2013). The observed gender differences are interesting. Given the association between BP and behavioral effects of FO observed by others (Harbild et al. 2013), we speculate that the effect of FO on BP may in part occur via the autonomic nervous system and might be related to a calming effect of FO in boys. In support of this hypothesis, our earlier infant study found that FO supplementation gave longer mean RR interval among boys (Lauritzen et al. 2008), which corresponds to a lower HR. However, in the present study, HR tended to increase with FO supplementation and higher HR was also observed among $2^{1 / 2}$-year-old boys whose mothers had been supplemented with FO during lactation (Larnkjaer et al. 2006).

The main strength of the present study is the long supplementation period and the inclusion of SNPs related to the proposed mechanisms of n-3 LCPUFA. The main limitation of the study is the relatively low number of study participants, especially with regards to the low number of subjects with minor alleles of the included SNPs. Our SNP modifying effects should, therefore, be considered explorative, and they need to be verified in studies with more participants. The baseline value of RBC n-3 LCPUFA was fairly high $(\sim 7.5 \mathrm{FA} \%)$ compared with other populations, and fish intake was somewhat higher in the FO group. This may have limited our ability to observe an effect of the FO intervention, also given that the metabolic outcomes were already low. We speculate that FO supplementation in a 
child population with a lower RBC n-3 LCPUFA status has more pronounced effects on metabolic outcomes. We did, however, observe differences in TAG and MAP after the intervention that must be ascribed the FO supplementation, as confirmed by the observed effect modifications by $P P A R G 2$ and $C O X 2$.

The consequences of lower plasma TAG and BP during the first years of life remain unknown. However, the fact that the effects of FO on metabolic markers in young children resemble those seen in adults is interesting and could indicate that the current intake of n-3 LCPUFA may be insufficient. Even though Danish infants and children have a relatively high intake of $n-3$ LCPUFA (median fish intake of $\sim 9 \mathrm{~g} /$ day in the present study) (Welch et al. 2002), they do not meet the recommendations of $\sim 20 \mathrm{~g} /$ day (extrapolated from the recommended 30-40 g/d for an adult with an energy intake of around $9 \mathrm{MJ} / \mathrm{d}$ to an energy intake of $3.8 \mathrm{MJ} / \mathrm{d}$ ).

In conclusion, our results confirm that n-3 LCPUFA reduces TAG and BP in early childhood and indicate that the effects are mediated via PPAR $\gamma 2$ and COX-2. We also found that FO reduced plasma glucose among PPARG2 heterozygotes. PPARG genotypes should therefore be considered in future studies examining effects of FO on HOMA.

Acknowledgments We gratefully thank all the infants and their families for their participation in EFiON, the biotechnicians: Inge Rasmussen and Vivian Anker and the students and scientific staff: Ditte Larsen, Ditte Aakjær, and Tine Launholt. Thanks to Axellus A/S for their kind donation of intervention oils. EFiON was supported by the Danish Council for Strategic Research, Programme Commission for Food and Health.

Conflict of interest Laurine B S Harsløf, Camilla T Damsgaard, Lars I Hellgren, Anders D Andersen, Ulla Vogel, and Lotte Lauritzen declare that they have no conflict of interest.

Ethical standard All procedures followed were in accordance with the ethical standards of the responsible committee on human experimentation (institutional and national) and with the Helsinki Declaration of 1975, as revised in 2000. Informed consent was obtained from all parents of the participants for being included in the study.

\section{References}

Abete I, Parra D, Crujeiras AB, Goyenechea E, Martinez JA (2008) Specific insulin sensitivity and leptin responses to a nutritional treatment of obesity via a combination of energy restriction and fatty fish intake. J Hum Nutr Diet 21:591-600

Ahmadian M, Suh JM, Hah N, Liddle C, Atkins AR, Downes M, Evans RM (2013) PPARgamma signaling and metabolism: the good, the bad and the future. Nat Med 19:557-566

Akinkuolie AO, Ngwa JS, Meigs JB, Djousse L (2011) Omega-3 polyunsaturated fatty acid and insulin sensitivity: a metaanalysis of randomized controlled trials. Clin Nutr 30:702-707

Andersen AD, Michaelsen KF, Hellgren LI, Trolle E, Lauritzen L (2011) A randomized controlled intervention with fish oil versus sunflower oil from 9 to 18 months of age: exploring changes in growth and skinfold thicknesses. Ped Res 70:368-374

Burdge GC, Calder PC (2005) Conversion of alpha-linolenic acid to longer-chain polyunsaturated fatty acids in human adults. Reprod Nutr Dev 45:581-597

Campa D, Zienolddiny S, Maggini V, Skaug V, Haugen A, Canzian F (2004) Association of a common polymorphism in the cyclooxygenase 2 gene with risk of non-small cell lung cancer. Carcinogenesis 25:229-235

Campbell F, Dickinson HO, Critchley JA, Ford GA, Bradburn M (2013) A systematic review of fish-oil supplements for the prevention and treatment of hypertension. Eur J Prev Cardiol 20:107-120

Chen X, Wang Y (2008) Tracking of blood pressure from childhood to adulthood: a systematic review and meta-regression analysis. Circulation 117:3171-3180

Cutfield WS, Jefferies CA, Jackson WE, Robinson EM, Hofman PL (2003) Evaluation of HOMA and QUICKI as measures of insulin sensitivity in prepubertal children. Pediatr Diabetes 4:119-125

Damsgaard CT, Schack-Nielsen L, Michaelsen KF, Fruekilde MB, Hels O, Lauritzen L (2006) Fish oil affects blood pressure and the plasma lipid profile in healthy Danish infants. J Nutr 136:94-99

Davidson MH (2006) Mechanisms for the hypotriglyceridemic effect of marine omega-3 fatty acids. Am J Cardiol 98:27-33

D’Orazio P, Burnett RW, Fogh-Andersen N, Jacobs E, Kuwa K, Kulpmann WR, Larsson L, Lewenstam A, Maas AH, Mager G, Naskalski JW, Okorodudu AO (2005) Approved IFCC recommendation on reporting results for blood glucose (abbreviated). Clin Chem 51:1573-1576

Egert S, Fobker M, Andersen G, Somoza V, Erbersdobler HF, Wahrburg U (2008) Effects of dietary alpha-linolenic acid, eicosapentaenoic acid or docosahexaenoic acid on parameters of glucose metabolism in healthy volunteers. Ann Nutr Metab 53:182-187

Forsyth JS, Willatts P, Agostoni C, Bissenden J, Casaer P, Boehm G (2003) Long chain polyunsaturated fatty acid supplementation in infant formula and blood pressure in later childhood: follow up of a randomised controlled trial. BMJ 326:953-957

Giacco R, Cuomo V, Vessby B, Uusitupa M, Hermansen K, Meyer BJ, Riccardi G, Rivellese AA (2007) Fish oil, insulin sensitivity, insulin secretion and glucose tolerance in healthy people: is there any effect of fish oil supplementation in relation to the type of background diet and habitual dietary intake of n-6 and n-3 fatty acids? Nutr Metab Cardiovasc Dis 17:572-580

Gondolf UH, Tetens I, Hills AP, Michaelsen KF, Trolle E (2012) Validation of a pre-coded food record for infants and young children. Eur J Clin Nutr 66:91-96

Harbild HL, Harslof LB, Christensen JH, Kannass KN, Lauritzen L (2013) Fish oil-supplementation from 9 to 12 months of age affects infant attention in a free-play test and is related to change in blood pressure. Prostaglandins Leukot Essent Fatty Acids 89:327-333

Harris WS (1997) n-3 fatty acids and serum lipoproteins: human studies. Am J Clin Nutr 65:1645S-1654S

Harslof LB, Larsen LH, Ritz C, Hellgren LI, Michaelsen KF, Vogel U, Lauritzen L (2013) FADS genotype and diet are important determinants of DHA status: a cross-sectional study in Danish infants. Am J Clin Nutr 97:1403-1410

Hartweg J, Perera R, Montori V, Dinneen S, Neil HA, Farmer A (2008) Omega-3 polyunsaturated fatty acids (PUFA) for type 2 diabetes mellitus. Cochrane Database Syst Rev (1) CD003205

Heinemann L (2010) Insulin assay standardization: leading to measures of insulin sensitivity and secretion for practical clinical care: response to Staten. Diabetes Care 33:e83

Larnkjaer A, Christensen JH, Michaelsen KF, Lauritzen L (2006) Maternal fish oil supplementation during lactation does not 
affect blood pressure, pulse wave velocity, or heart rate variability in 2.5-y-old children. J Nutr 136:1539-1544

Lauritzen L, Jorgensen MH, Mikkelsen TB, Skovgaard M, Straarup EM, Olsen SF, Hoy CE, Michaelsen KF (2004) Maternal fish oil supplementation in lactation: effect on visual acuity and n-3 fatty acid content of infant erythrocytes. Lipids 39:195-206

Lauritzen L, Christensen JH, Damsgaard CT, Michaelsen KF (2008) The effect of fish oil supplementation on heart rate in healthy Danish infants. Pediatr Res 64:610-614

Lindi V, Schwab U, Louheranta A, Laakso M, Vessby B, Hermansen K, Storlien L, Riccardi G, Rivellese A (2003) Impact of the Pro12Ala polymorphism of the PPAR-gamma2 gene on serum triacylglycerol response to n-3 fatty acid supplementation. Mol Genet Metab 79:52-60

Marquardt A, Stohr H, White K, Weber BH (2000) cDNA cloning, genomic structure, and chromosomal localization of three members of the human fatty acid desaturase family. Genomics 66:175-183

Masugi J, Tamori Y, Mori H, Koike T, Kasuga M (2000) Inhibitory effect of a proline-to-alanine substitution at codon 12 of peroxisome proliferator-activated receptor-gamma 2 on thiazolidinedione-induced adipogenesis. Biochem Biophys Res Commun 268:178-182

Miller SA, Dykes DD, Polesky HF (1988) A simple salting out procedure for extracting DNA from human nucleated cells. Nucleic Acids Res 16:1215

Nakamura MT, Nara TY (2004) Structure, function, and dietary regulation of delta6, delta5, and delta9 desaturases. Ann Rev Nutr 24:345-376

Pedersen MH, Molgaard C, Hellgren LI, Lauritzen L (2010) Effects of fish oil supplementation on markers of the metabolic syndrome. J Pediatr 157:395-400

Poudyal H, Panchal SK, Diwan V, Brown L (2011) Omega-3 fatty acids and metabolic syndrome: effects and emerging mechanisms of action. Prog Lipid Res 50:372-387

Ramel A, Martinez A, Kiely M, Morais G, Bandarra NM, Thorsdottir I (2008) Beneficial effects of long-chain n-3 fatty acids included in an energy-restricted diet on insulin resistance in overweight and obese European young adults. Diabetologia 51:1261-1268
Sanak M, Szczeklik W, Szczeklik A (2005) Association of COX-2 gene haplotypes with prostaglandins production in bronchial asthma. J Allergy Clin Immunol 116:221-223

Stumvoll M, Haring H (2002) The peroxisome proliferator-activated receptor-gamma2 Pro12Ala polymorphism. Diabetes 51:23412347

Tapiero H, Ba GN, Couvreur P, Tew KD (2002) Polyunsaturated fatty acids (PUFA) and eicosanoids in human health and pathologies. Biomed Pharmacother 56:215-222

Tontonoz P, Spiegelman BM (2008) Fat and beyond: the diverse biology of PPARgamma. Annu Rev Biochem 77:289-312

Vogel U, Christensen J, Nexo BA, Wallin H, Friis S, Tjonneland A (2007a) Peroxisome proliferator-activated receptor-gamma2 Pro12Ala, interaction with alcohol intake and NSAID use, in relation to risk of breast cancer in a prospective study of Danes. Carcinogenesis 28:427-434

Vogel U, Christensen J, Wallin H, Friis S, Nexo BA, Tjonneland A (2007b) Polymorphisms in COX-2, NSAID use and risk of basal cell carcinoma in a prospective study of Danes. Mutat Res 617:138-146

Welch AA, Lund E, Amiano P, Dorronsoro M, Brustad M, Kumle M, Rodriguez M, Lasheras C, Janzon L, Jansson J, Luben R, Spencer EA, Overvad K, Tjonneland A, Clavel-Chapelon F, Linseisen J, Klipstein-Grobusch K, Benetou V, Zavitsanos X, Tumino R, Galasso R, Bueno-De-Mesquita HB, Ocke MC, Charrondiere UR, Slimani N (2002) Variability of fish consumption within the 10 European countries participating in the European Investigation into Cancer and Nutrition (EPIC) study. Public Health Nutr 5:1273-1285

Ylonen SK, Salminen I, Lyssenko V, Virtanen SM, Groop L, Aro A, Saloranta C (2008) The Pro12Ala polymorphism of the PPARgamma2 gene affects associations of fish intake and marine n-3 fatty acids with glucose metabolism. Eur J Clin Nutr 62:1432-1439

Zhang X, Miao X, Tan W, Ning B, Liu Z, Hong Y, Song W, Guo Y, Zhang X, Shen Y, Qiang B, Kadlubar FF, Lin D (2005) Identification of functional genetic variants in cyclooxygenase-2 and their association with risk of esophageal cancer. Gastroenterology 129:565-576 